\title{
Einblick in den Medizinjournalismus Journalistenschüler besuchen die Einblick-Redaktion des DKFZ
}

Das Deutsche Krebsforschungszentrum (DKFZ) in Heidelberg zählt international zu den bedeutendsten Einrichtungen zur Erforschung von Krebsursachen, -risiken und -therapien. Die Kommunikation zwischen der Forschungseinrichtung mit seinen über 1000 Wissenschaftlern und der Öffentlichkeit läuft häufig über Wissenschaftsjournalisten. Da Gesundheitsthemen in der heutigen Wissensgesellschaft immer mehr an Bedeutung gewinnen, setzt sich das DKFZ auch für die Förderung des medizinjournalistischen Nachwuchses ein.

Das Interesse der Öffentlichkeit an wissenschaftlichen Themen ist in den letzten 20 Jahren stetig und rasant angestiegen. Während bis in die frühen 90er Jahre fast ausschließlich Fachpublikationen über Themen wie Gesundheit, Klimawandel oder Kernenergie informierten, kommt heute kaum mehr eine Regionalzeitung ohne Wissenschaftsressorts aus. Das Leserinteresse an medizinischen Themen ist dabei besonders hoch. Nicht selten bilden heute wichtige Neuigkeiten aus der Medizin die Hauptnachricht des Tages. So auch die Ende Oktober letzten Jahres ausgegebene Meldung der Weltgesundheitsorganisation, dass der Verzehr von Wurst und Schinken das Krebsrisiko erhöhe.

„Eine ganze Woche hat mein Telefon ununterbrochen geklingelt“, verrät Stefanie Seltmann, Leiterin der Stabsstelle Presse- und Öffentlichkeitsarbeit des DKFZ bei einem Treffen mit Schülern der Freien Journalistenschule (FJS). ,Verbraucher sind besorgt, die Fleischindustrie entsetzt, Politiker in Erklärungsnot. Und alle Medien wollen berichten. Jeder auf seine Weise. Von den großen TV-Anstalten über die BoulevardMedien bis hin zu Regionalzeitungen.“

Die Pressestelle des DKFZ versteht sich als Mittler zwischen der biomedizinischen Forschungsanstalt und der Öffentlichkeit. Ihre Aufgabe ist es, die Arbeit der fast 3000 Mitarbeiter nach außen zu repräsentieren sowie die gesamte interne und externe Kommunikation zu managen. Das gilt für Riesenevents, wie beispielsweise im vergangenen April die Planung und Begleitung des Besuches von Angela Merkel zum 50-jährigen Jubiläum der Forschungsanstalt, genauso wie für Gespräche mit angehenden Wissenschaftsjournalisten. Auch Seltmann ist als Chefredakteurin des hauseigenen Magazins Einblick, das 2-3-mal jährlich mit einer Auflage von 13000 erscheint und über Krebs, Krebsforschung und Krebsmedizin informiert, Wissenschaftsjournalistin.

\section{Medizin und Journalismus}

historisch verwurzelt

„Unsere Leser sind zwischen 18 und 95 Jahre alt. Dazu gehören vor allem Patien-

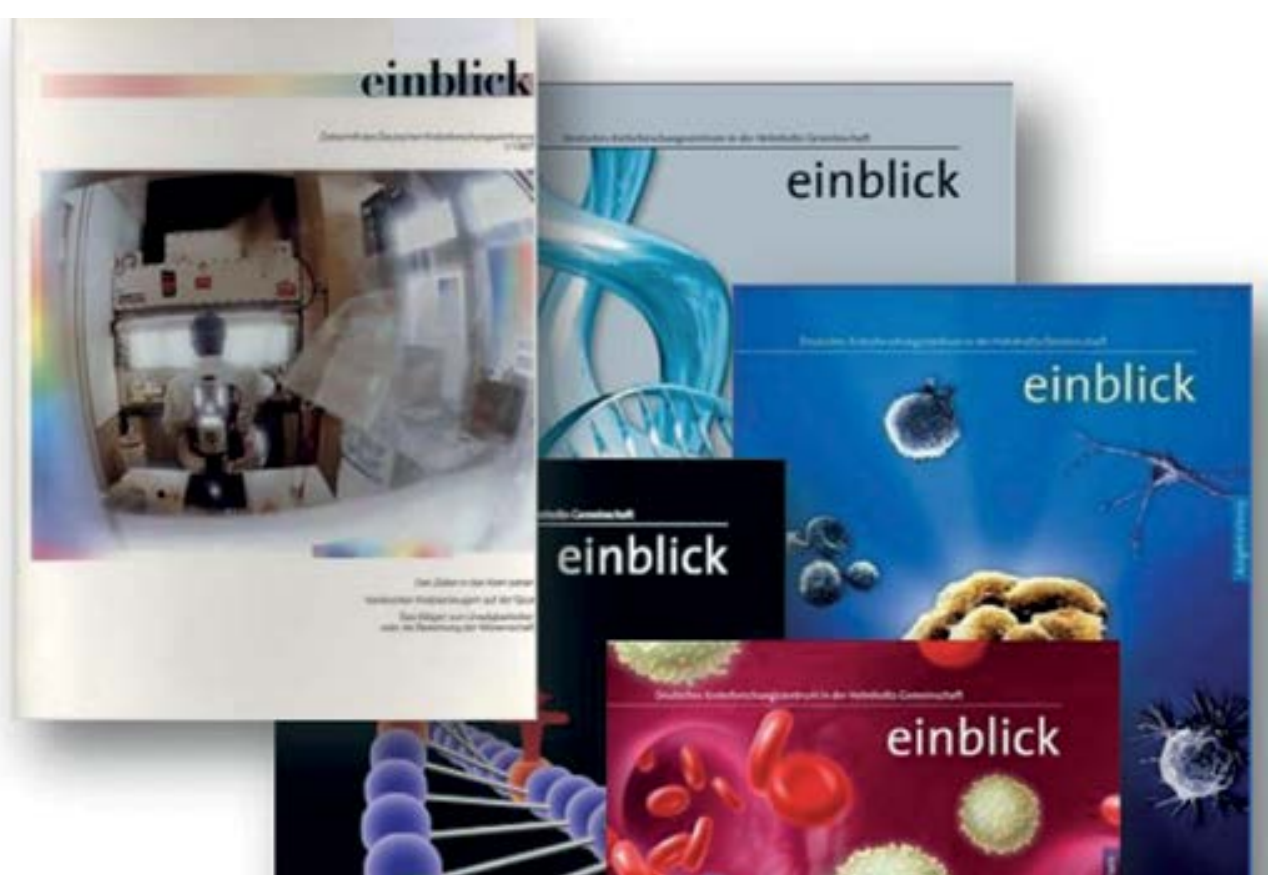

ten und Angehörige“, sagt Seltmann. „Es ist die große Kunst der Medizin- und Wissenschaftsjournalisten, neueste Forschungserkenntnisse zielgruppengerecht aufzubereiten und informativ, spannend und unterhaltsam zu vermitteln.“

Medizin und Journalismus sind 2 Disziplinen, die schon seit der Antike Hand in Hand gehen und sich im Laufe der Menschheitsgeschichte immer wieder gegenseitig beeinflussten und voneinander abhängig waren. Denn immer war es auch Anspruch von Medizinern, ihr Wissen zu verbreiten und Bedürfnis der Kranken, sich über Heilungsmethoden zu informieren. Der Journalismus war Schnittstelle, ob als Einblattdruck im 16. Jahrhundert oder als Gesundheitsblog in der heutigen digitalen Welt. Es erstaunt kaum, dass die erste wissenschaftliche Erörterung des Pressewesens aus der Feder eines Arztes stammte. Tobias Peucer legte seine Dissertation De Relationibus Novellis (Über Zeitungsberichte) 1690 an der Universität Leipzig ab.

Anders als in den USA, wo Journalisten, die explizit über medizinische Themen schreiben, sich als „medical editors“ oder „medical writers“ bezeichnen, verstehen sich ihre deutschen Kollegen allgemeiner als Wissenschaftsjournalisten, die sich flexibel in unterschiedliche Forschungsbereiche einarbeiten können.

Frank Schubert, Redakteur bei den Wissenschaftsmagazinen Spektrum der Wissenschaft und Gehirn und Geist, klärt die FJS-Schüler am DKFZ in einem Vortrag auf: „Eine der wichtigsten Herausforderungen für Wissenschaftsjournalisten ist es, wissenschaftliche Themen einem breiten Publikum zugänglich zu machen.“ Dafür seien Redaktionen auf Mitarbeiter angewiesen, die in beiden Welten - Wissenschaft und Journalismus - zu Hause sind. „Unsere Redakteure haben fast alle wissenschaftlich promoviert und haben durchweg jahrelange Erfahrung als Journalisten“, sagt der Biophysiker.

\section{Experten mit journalistischen Skills}

Allrounder haben heute kaum Chancen auf eine Festanstellung als Journalist. Gefragt sind Experten mit einer journalisti- 
schen Zusatzausbildung. Die universitäre Landschaft hat reagiert, mittlerweile haben sich in Dortmund und Darmstadt 2 wissenschaftsjournalistische Studiengänge etabliert. Hier werden neben speziellen Fachwissenschaften die Grundlagen des Journalismus gelehrt.

„Wer bereits ein Fachstudium abgeschlossen hat, bekommt bei uns per Fernstudium die Möglichkeit, sich zusätzlich zum Wissenschaftsjournalisten ausbilden zu lassen. In 12 aufeinanderfolgenden Modulen erwerben die Teilnehmer das Rüstzeug für einen guten, verlagsunabhängigen Fachjournalismus“, sagt René Teichmann, Leiter der FJS. Seine Schüler wollten entweder neben ihrem Beruf als Arzt, Jurist oder Historiker publizieren oder sich hauptberuflich als Wissenschaftsjournalist betätigen. Regelmäßige Exkursionen zu verschiedenen wissenschaftsjournalistischen Redaktionen seien dabei wichtige, zusätzliche Orientierungspunkte.

Das bestätigt auch Anne Glöggler bei ihrem Besuch der Redaktion Einblick. Die promovierte Ernährungswissenschaftlerin ist Geschäftsführerin eines Verlags für Medizin und Naturwissenschaften. „Wir bringen im Jahr etwa 15 medizinische Sach- und Fachbücher und 2 Zeitschriften heraus. Unsere Autoren sind Ärzte und Fachkräfte aus verschiedenen medizinischen und pflegerischen Bereichen.“

Oftmals sei es nicht leicht, Medizinern zu erklären, dass ein Patientenratgeber sehr viel einfacher geschrieben werden müsse als eine Fachpublikation. „Da ist Fingerspitzengefühl gefragt“, sagt Glöggler. „Durch das Studium an der FJS habe ich mehr Standing, kann vieles besser begründen. Ich profitiere aber auch ungemein von Modulen wie Presserecht, Öffentlichkeitsarbeit und Fotojournalismus."

Ähnlich begeistert vom Besuch beim DKFZ zeigte sich Sören Feddersen. „Ich habe gerade erst an der FJS angefangen. Öffentlichkeitsarbeit ist auch Teil des Lehrplans und hier habe ich erfahren, wie PR-Arbeit in der Praxis aussieht. Für ein Praktikum habe ich mich dann auch gleich beworben“, sagt der studierte Lebensmittelchemiker. „Ich kann mir sehr gut vorstellen, nach dem Studium im Medizin-PR-Bereich zu arbeiten. Heute gibt es da viele Herausforderungen. Neben Mitarbeiterkommunikation, Marketing und Presseservice spielen Auftritte in sozialen Medien eine immer größere Rolle.“

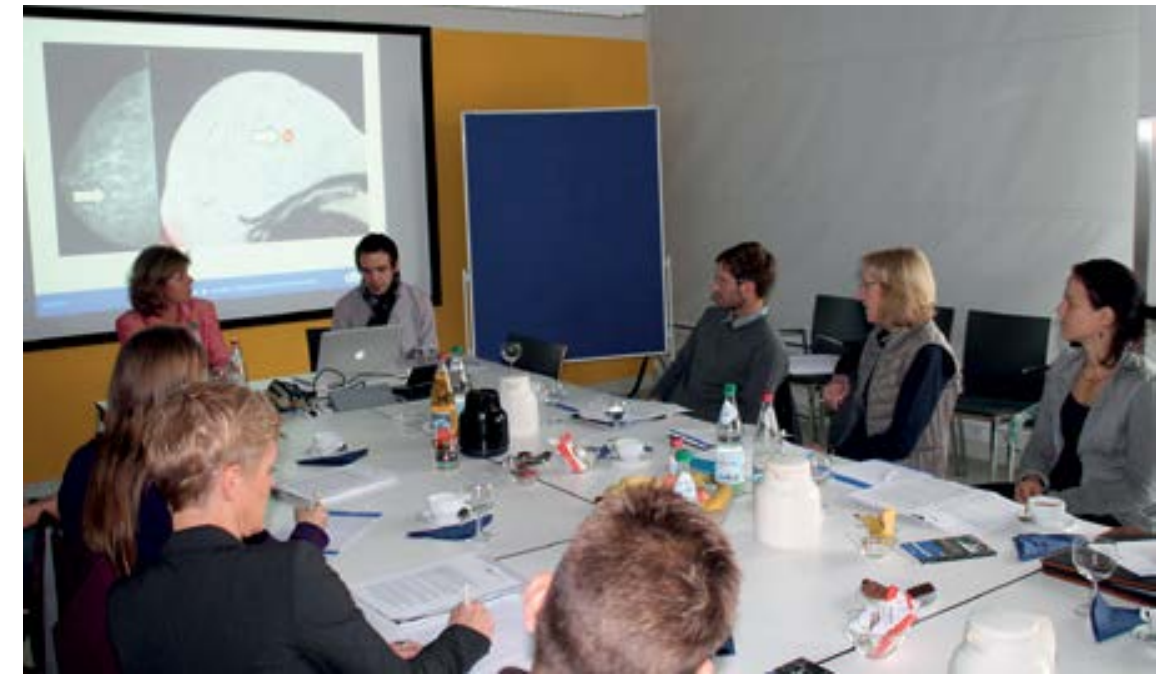

Abb. 1 Journalistenschüler bei einer Pressekonferenz mit PR-Chefin Stefanie Seltmann und DKFZ-Forscher Sebastian Bickelhaupt.

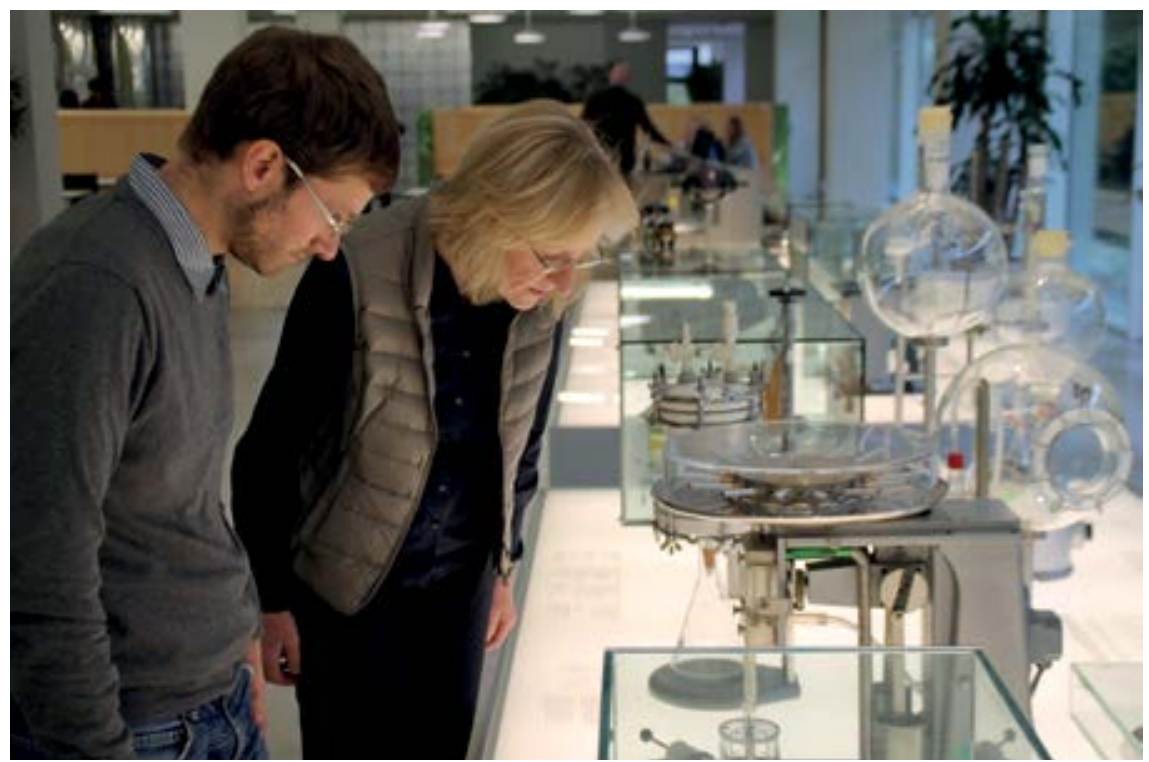

Abb. 2 FJS-Schüler Sören Feddersen und Anne Glöggler inspizieren Forschungsapparaturen im DKFZ-Museum.

\section{Wissenschaftskommunikation trainieren}

Zum Abschluss des Besuches der Journalistenschüler hat sich Pressesprecherin Seltmann etwas besonders Praktisches ausgedacht: eine Live-Pressekonferenz als Planspiel. Dazu eingeladen ist der DKFZ-Wissenschaftler Sebastian Bickelhaupt, der soeben eine Studie zur Früherkennung von Brustkrebs durch Magnetresonanz-Tomografie abgeschlossen hat. Eine gute Übung auch für den Radiologen, denn die regulären Pressekonferenzen stehen erst noch an. Die angehenden Wissenschaftsjournalisten der FJS aber haben die brandaktuelle Pressemitteilung schon penibel auseinandergenommen und löchern den Mediziner mit Fra- gen, als wären die Ergebnisse seiner Studie die mediale Hauptnachricht von morgen. Das Vertrauen in eine professionelle wissenschaftsjournalistische Vermittlung hat er zumindest an diesem Tag gewinnen können.

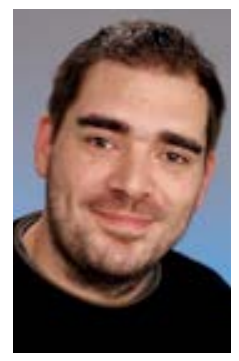

Korrespondenz Dr. phil. Christian Hardinghaus Historiker, Medien-und Literaturwissenschaftler Krahnstraße 30 49074 Osnabrück Fax: 03212/4273464 E-Mail: presse@harding haus.info 